\title{
Spatial and Temporal Scales of Neuronal Correlation in Visual Area V4
}

\author{
Matthew A. Smith ${ }^{1,2}$ and Marc A. Sommer ${ }^{2,3}$ \\ ${ }^{1}$ Department of Ophthalmology and ${ }^{2}$ Department of Neuroscience and the Center for the Neural Basis of Cognition, University of Pittsburgh, Pittsburgh, \\ Pennsylvania 15213, and ${ }^{3}$ Department of Biomedical Engineering, Center for Cognitive Neuroscience, and Duke Institute for Brain Sciences, Duke \\ University, Durham, North Carolina 27708
}

The spiking activity of nearby cortical neurons is correlated on both short and long time scales. Understanding this shared variability in firing patterns is critical for appreciating the representation of sensory stimuli in ensembles of neurons, the coincident influences of neurons on common targets, and the functional implications of microcircuitry. Our knowledge about neuronal correlations, however, derives largely from experiments that used different recording methods, analysis techniques, and cortical regions. Here we studied the structure of neuronal correlation in area V4 of alert macaques using recording and analysis procedures designed to match those used previously in primary visual cortex (V1), the major input to V4. We found that the spatial and temporal properties of correlations in V4 were remarkably similar to those of V1, with two notable differences: correlated variability in V4 was approximately one-third the magnitude of that in V1 and synchrony in V4 was less temporally precise than in V1. In both areas, spontaneous activity (measured during fixation while viewing a blank screen) was approximately twice as correlated as visual-evoked activity. The results provide a foundation for understanding how the structure of neuronal correlation differs among brain regions and stages in cortical processing and suggest that it is likely governed by features of neuronal circuits that are shared across the visual cortex.

\section{Introduction}

The spiking activity of pairs of nearby cortical neurons is correlated. The correlations occur over a range of time scales, from slow fluctuations in responsivity over periods of hundreds of milliseconds (correlated variability) to coordinated firing over a range of a few milliseconds (synchrony). Both forms of correlation have been implicated as important for population coding. Correlated variability is directly related to the amount of information present in a population of neurons (Zohary et al., 1994; Shadlen and Newsome, 1998; Abbott and Dayan, 1999; Averbeck et al., 2006) and the particular structure of the correlation-its dependence on tuning similarity, neuronal separation, and other factors-determines the magnitude of the information content (Averbeck et al., 2006). Synchrony has been associated with a number of visual and cognitive phenomenon, including the binding of visual objects, attention, and arousal (Gray, 1999; Shadlen and Movshon, 1999). Given the broad range of effects for which correlations may play a role, it is apparent that a more

\footnotetext{
Received 0ct. 9, 2012; revised Jan. 26, 2013; accepted Feb. 18, 2013.

Author contributions: M.A. Smith and M.A. Sommer designed research; M.A. Smith performed research; M.A. Smith analyzed data; M.A. Smith and M.A. Sommer wrote the paper.

This work was supported by the National Eye Institute (NEI Grant EY018894 to M.A. Smith and Grant EY017592 to M.A. Sommer), the Eye and Ear Foundation of Pittsburgh, and Research to Prevent Blindness (Unrestricted Grant and Career Development Award to M.A. Smith). Technical support was provided by NEl Core Grant P30 EY08098. We thank Karen McCracken and Cory Willis for technical assistance, Ryan Kelly and Matt Harrison for providing software, and Patrick Mayo and Adam Snyder for comments on a previous draft of this manuscript.

Correspondence should be addressed to Matthew A. Smith, Department of Ophthalmology, University of Pittsburgh, 203 Lothrop St, Eye and Ear Institute, Room 914, Pittsburgh, PA 15213. E-mail: smithma@pitt.edu.

DOI:10.1523/JNEUROSCI.4782-12.2013

Copyright $\odot 2013$ the authors $\quad 0270-6474 / 13 / 335422-11 \$ 15.00 / 0$
}

complete understanding of the structure of correlated activity is needed.

Correlated variability and synchrony have been measured by a number of studies of primary visual cortex, or V1 (for review, see Cohen and Kohn, 2011). This work was performed in a number of laboratories with different stimuli, recording methods, and analysis procedures, leading to a fragmented picture of the structure of correlation. In research performed by an author of the present study, the structure of neuronal correlation in V1 was found to depend on the distance between neurons, their tuning similarity, and the temporal scale (Smith and Kohn, 2008). Outside of V1, a small number of studies have measured correlated variability in areas such as V4 (Cohen and Maunsell, 2009; Mitchell et al., 2009) and MT (Zohary et al., 1994; Bair et al., 2001; Cohen and Newsome, 2009; Huang and Lisberger, 2009). These studies tended to focus on one time scale and they used diverse recording techniques and visual stimuli.

Our goal was to evaluate how neuronal correlations compare between separate, but closely linked, visual cortical regions. We used 100-electrode arrays (Fig. 1A) to record from populations of neurons in macaque area $\mathrm{V} 4$, which is proposed to play a role in a number of midlevel visual processes, including spatial and feature-based attention, figure-ground segregation, color and brightness perception, and shape representation (for review, see Roe et al., 2012). V4 receives its primary input from V1, so we investigated whether the prominent features of neuronal correlation that we observed in V1 (Smith and Kohn, 2008), namely the dependence of correlation on distance, tuning similarity, and time scale, were also present in V4. We used the same stimuli and analysis methods as we had in V1 to make as close a comparison 

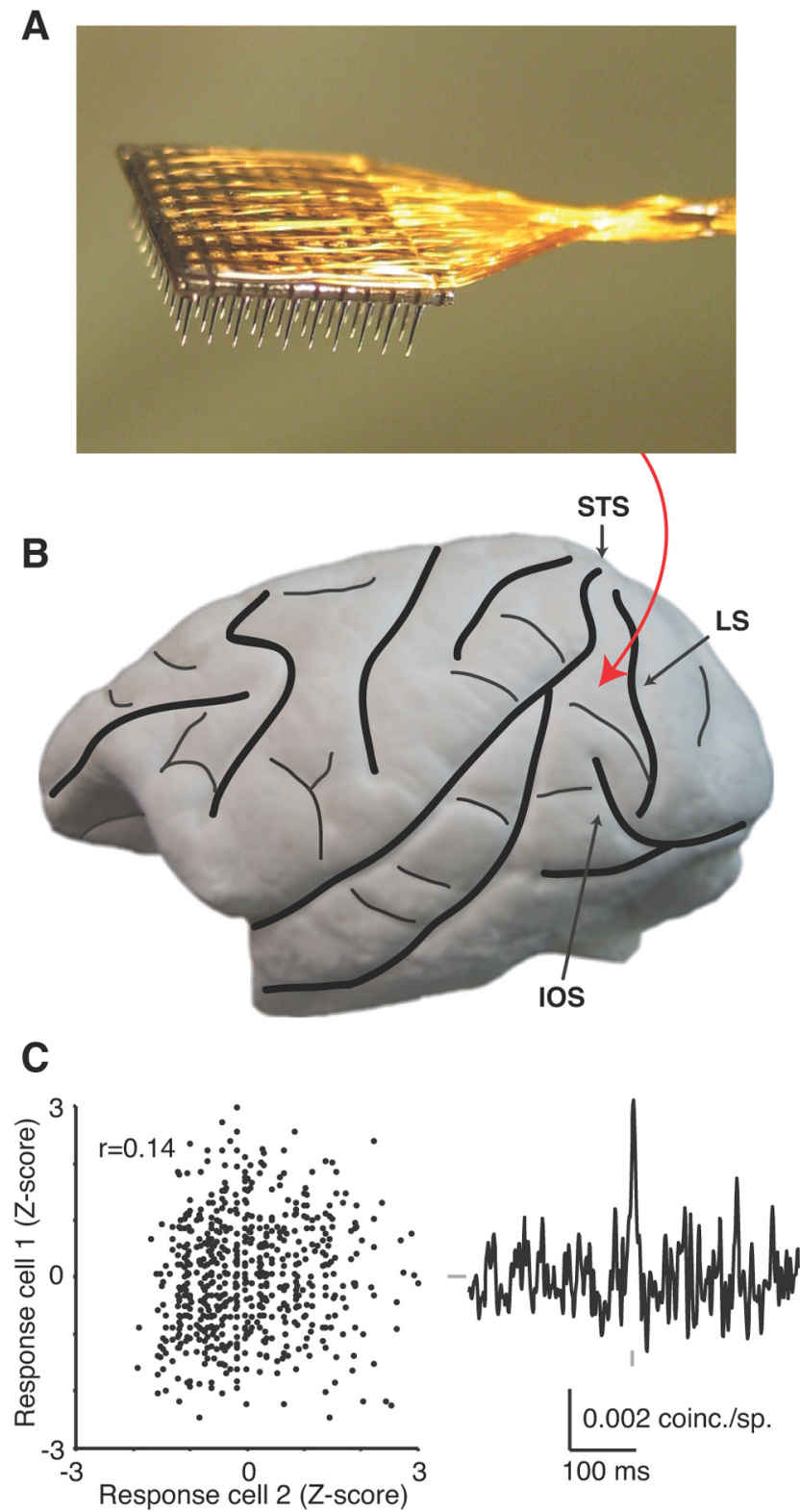

Figure 1. Experimental methods. $A$, Photograph of the array, consisting of a $10 \times 10$ grid of silicone microelectrodes with $400 \mu \mathrm{m}$ spacing and $1.0 \mathrm{~mm}$ length. $\boldsymbol{B}$, Lateral view of the macaque brain with major sulci indicated with thick black lines and minor vessels and sulci with thin black lines. The rough position of the inserted arrays, anterior to the lunate sulcus (LS), posterior to the superior temporal sulcus (STS), and medial/dorsal to the inferior occipital sulcus (IOS), is indicated with the red arrow. C, For a single pair of V4 neurons, the plot on the left shows the $z$-scored spike counts of one neuron on all of the trials (of all 12 stimuli) plotted as a function of the same metric in a second neuron. The Pearson's correlation of these data points was 0.14 $(p<0.0001)$. For the same pair of neurons, the jitter-corrected CCG is shown (with a jitter window of $50 \mathrm{~ms}$ ). The vertical gray tick represents zero time lag and the horizontal gray tick represents 0 coinc./sp. Scale bars are shown below the CCG.

as possible. We found that correlations among V4 neurons were quite similar to those found in V1, except for decreased magnitudes and a broader relationship between time scales and distance. We conclude that the structure of neuronal correlation is governed primarily by features of neuronal circuits that are shared across the visual cortex.

\section{Materials and Methods}

Surgical preparation. Two adult male rhesus macaque monkeys (Macaca mulatta) were surgically prepared in aseptic conditions under isofluo- rothane anesthesia. In one animal (Monkey D), we implanted a scleral search coil for eye position monitoring (Judge et al., 1980) and a post for immobilizing the head during experiments (see Sommer and Wurtz, 2000), with an acrylic implant and titanium screws affixing the eye coil connector and head post to the skull. In the other animal (Monkey B), we implanted only a titanium head post held to the skull with titanium screws. All procedures were approved by the Institutional Animal Care and Use Committee of the University of Pittsburgh and were in compliance with the guidelines set forth in the National Institutes of Health's Guide for the Care and Use of Laboratory Animals.

In addition, we implanted a 100-electrode "Utah" array (Blackrock Microsystems) in visual area V4 of each animal. The array consisted of a $10 \times 10$ grid of silicon microelectrodes (each $1 \mathrm{~mm}$ in length) spaced 400 $\mu \mathrm{m}$ apart (Fig. $1 A$ ). The implantation was performed in the same surgery as the head post and eye coil for Monkey D, but in a separate surgery for Monkey B. Area V4 was located with guidance from stereotaxic coordinates (Horsley-Clarke: the intersection of $5 \mathrm{~mm}$ posterior and $12-15 \mathrm{~mm}$ dorsal with the surface of the skull) and then identified directly by performing a craniotomy and durotomy and visualizing the lunate and superior temporal sulci. The arrays were implanted using a pneumatic insertion device (Rousche and Normann, 1992) on the prelunate gyrus just dorsal to the inferior occipital sulcus (Fig. 1B). This resulted in neuronal receptive fields centered $\sim 1.5^{\circ}$ from the fovea for Monkey $B$ and $3^{\circ}$ for Monkey D.

Data collection. Signals from each microelectrode in the array were amplified and band-pass filtered $(250-7.5 \mathrm{kHz})$ by a Cerebus system (Blackrock Microsystems). For each electrode in the array, waveform segments that exceeded a threshold (periodically adjusted using a multiple of the rms noise on each channel) were digitized $(30 \mathrm{kHz})$ and stored for offline analysis and sorting. Waveform segments were sorted with an automated algorithm that clustered similarly shaped waveforms using a competitive mixture decomposition method (Shoham et al., 2003). We manually refined the output of this algorithm with custom timeamplitude window discrimination software (written in MATLAB; MathWorks) for each electrode, taking into account the shape of the waveform and the distribution of interspike intervals.

Following the offline sorting procedure, we computed the signal-tonoise ratio (SNR) of each candidate unit as the ratio of the average waveform amplitude to the SD of the waveform noise (Nordhausen et al., 1996; Suner et al., 2005; Kelly et al., 2007). Candidates that fell below an SNR of 2.75 were discarded as probable multiunit recordings. We also eliminated neurons for which the best visual stimulus (gratings as described below) did not evoke a response of at least 2 spikes/s (33 units removed). The remaining candidate waveforms (113 units) were deemed to be single neurons of sufficient quality and visual responsiveness to warrant further analysis.

In Monkey D, we monitored eye position with a scleral search coil system (Riverbend Instruments). In Monkey B, we used an infrared tracking system (ISCAN). In each case, the animals were required to maintain fixation within $0.5^{\circ}$ of a small spot. Eye movements were analyzed online by the behavioral control software to ensure fixation within the required window, but these data were not saved due to a firmware error.

Visual stimulation and behavioral task. Visual stimuli were indistinguishable for the two animals except for their sizes and positions that were matched to the receptive fields studied in each monkey. For Monkey $\mathrm{B}$, we generated the stimuli using the REX real-time system (Hays et al., 1982), whereas for Monkey D we used custom software written in MATLAB using the Psychophysics Toolbox extensions (Brainard, 1997; Kleiner et al., 2007). All stimuli were displayed on a CRT monitor with a resolution of $1024 \times 768$ pixels and a temporal refresh of $100 \mathrm{~Hz}$ viewed at a distance of $57 \mathrm{~cm}$. We used lookup tables to correct for nonlinearities in the relation between input voltage and phosphor luminance in the monitor. The mean luminance of the display was $\sim 40 \mathrm{~cd} / \mathrm{m}^{2}$. All stimuli were presented in a circular aperture surrounded by a gray field of average luminance. We mapped the spatial receptive fields of each channel by presenting small, drifting gratings at a range of spatial positions. We centered our stimuli on the aggregate receptive field of the recorded units. 
An animal was required to fixate a $0.15^{\circ}$ blue dot for $100 \mathrm{~ms}$ to begin a trial. A drifting sinusoidal grating stimulus was then presented for 1000 ms. After the last video frame containing the stimulus and fixation spot, the animal was required to make a saccade to a small dot randomly positioned along a circle $8^{\circ}$ from fixation. If the animal left the central fixation window within $300 \mathrm{~ms}$ and made a saccade to the target window, it received a liquid reward. We presented full-contrast drifting sinusoidal gratings at 12 orientations spaced equally $\left(30^{\circ}\right)$. The spatial frequency $(1.3 \mathrm{cpd})$ and temporal frequency $(6.25 \mathrm{~Hz})$ values were chosen to match the values we used in a previous experiment in V1 neurons (Smith and Kohn, 2008). The position and size $\left(2.5^{\circ}\right.$ in Monkey B and $5^{\circ}$ in Monkey D) of the grating were sufficient to cover the receptive fields of all the neurons. Stimulus orientation was block randomized, and blocks were repeated 61 times for Monkey B and 80 times for Monkey D.

We also collected a block of data in which the animal was required to perform the same task without the grating present. Therefore, these data represented a $1000 \mathrm{~ms}$ period of spontaneous activity. In Monkey B, 301 spontaneous trials were collected after the grating data. In Monkey D, 200 spontaneous trials were collected before the grating data.

The data presented here represent all of the neurons recorded from a single behavioral session from each animal (the first day of recording for this paradigm). The same data collection procedures were used over the course of several days in each animal, but we chose to be conservative and not present data from successive days or pool data across days. We considered this important because uncertainty in the identity of single units or multiunit clusters recorded in successive days would almost certainly result in false overestimates of the number of uniquely recorded neuron pairs. However, analyses of the data in later days and analyses of the data pooled across days produced results that were equivalent to the results reported here for all tests.

Correlation analyses. The methods used to characterize correlation were presented in detail in two previous publications (Kohn and Smith, 2005; Smith and Kohn, 2008). Here, we describe the measures in brief. The spike count correlation, or $r_{\mathrm{sc}}$, quantifies the correlated variability as discussed in the Introduction. It is the Pearson correlation coefficient of the evoked spike counts of a pair of neurons in response to repeated presentations of the same stimulus. The $r_{\mathrm{sc}}$ captures the shared trial-totrial variability of firing rates averaged over the full $1000 \mathrm{~ms}$ stimulus presentation. For all trials of each stimulus orientation, we normalized each neuron's responses to a mean of zero and unit variance ( $z$-score) and calculated $r_{\mathrm{sc}}$ between neuronal pairs after combining responses to all stimulus orientations (Fig. 1C, left).

As a measure of the relative spike timing or synchrony of two neurons at a millisecond time scale, we computed the spike train crosscorrelogram (CCG; Perkel et al., 1967). We normalized the CCG by the geometric mean spike rate of the pair of neurons. This is the most commonly used normalization, the results of which are most easily compared with $r_{\text {sc }}$ (Kohn and Smith, 2005). A normalized CCG was computed for each of the 12 stimulus orientations and then smoothed with a $5 \mathrm{~ms}$ kernel ( $\left[\begin{array}{lllll}0.05 & 0.25 & 0.40 & 0.25 & 0.05\end{array}\right]$ ). We then took the mean value at each time lag across the CCGs to produce an average CCG for each pair (Fig. $1 C$, right).

We calculated CCGs with two types of correction. The standard shuffle-corrected CCG was created by subtracting the expected value of all possible CCGs produced using the same dataset but with nonsimultaneous trial pairings. This method removes correlations locked to the stimulus, but results in a CCG that reflects correlation on a range of time scales. We also used a jitter correction method (Amarasingham et al., 2012) to remove slow temporal correlations and stimulus locked correlations. This is created by subtracting the expected value of CCGs produced from a resampled version of the original dataset with the spike times randomly perturbed or jittered within a small window of time (the jitter window). Details on this method have been described previously (Smith and Kohn, 2008). We used a jitter window of $50 \mathrm{~ms}$ for most of the analysis shown, with the exception of the data shown in Figure 6, in which the jitter window was varied as described.

We calculated the tuning similarity of each pair of neurons as the Pearson's correlation between the mean responses of each cell to all 12 orientations $\left(r_{\text {signal }}\right)$. For neurons with similar orientation tuning, $r_{\text {signal }}$ is near 1 ; for neurons with dissimilar tuning, $r_{\text {signal }}$ approaches -1 .

As a measure of correlation on a time scale greater than the length of a single trial, we calculated the trial cross-covariance (TCC) following the procedure of Bair et al. (2001). Simply put, this is the cross-correlation of the $z$-scored spike counts for a pair of neurons across all trials. For a trial lag of zero, the TCC is equal to $r_{\mathrm{sc}}$. If the correlation between a pair of neurons arises only from time scales shorter than the duration of a trial, then the TCC will consist of an isolated peak at zero trial lag with all other values approaching zero.

Using the TCC, we estimated the long-term component of $r_{\text {sc }}$ (longterm correlation or $r_{1 \mathrm{t}}$ ). This was done by replacing the value of the TCC at zero with the average of each immediate neighbor. We then convolved the TCC with a Gaussian $(\mathrm{SD}=4$ trials $)$ and determined the new value at zero $\left(r_{1 t}\right)$.

Although $r_{\mathrm{sc}}$ is typically computed over the full length of the trial, correlation can arise on various time scales. To assess the contribution of effects on these time scales, we also computed a metric known as $r_{\text {ccg }}$ (introduced by Bair et al., 2001). This is the integral of the shufflecorrected CCG divided by the geometric mean area of the autocorrelograms of the pair of neurons. When calculated over the full trial duration, $r_{\text {ccg }}$ is equal to $r_{\mathrm{sc}}$. In shorter windows of integration, $r_{\mathrm{ccg}}$ is useful for revealing the magnitude of correlated variability on that time scale.

All indications of variation in the graphs and text are SEM. The statistical significance of all results was evaluated with two-tailed $t$ tests unless otherwise noted. All values of $r$ indicate Pearson's correlation unless otherwise noted. Significance of Pearson's correlation values was assessed after applying the Fisher $Z$-transform to the data.

\section{Results}

We measured pairwise correlations among all possible pairs, excluding pairings on the same electrode, of 113 neurons recorded from arrays implanted in area V4 of two macaque monkeys (Fig. 1). This resulted in 3240 total pairs (1818 from one animal and 1422 from the other).

\section{Correlated variability}

Pairs of neurons in visual cortex, including areas V1 and V4, exhibit shared trial-to-trial fluctuations in response strength (Kohn and Smith, 2005; Smith and Kohn, 2008; Cohen and Maunsell, 2009; Mitchell et al., 2009). Spike count correlation $\left(r_{\mathrm{sc}}\right)$ measures this correlated variability using the Pearson correlation of the evoked spike counts of two simultaneously recorded neurons over the entire $1000 \mathrm{~ms}$ stimulus period. Across all 3240 pairs of neurons recorded from both animals, the average $r_{\mathrm{sc}}$ was $0.058 \pm 0.001$ (0.063 in Monkey B and 0.053 in Monkey D), significantly greater than zero $(p<0.0001)$ and similar to correlation levels found in two previous studies of V4 activity (Cohen and Maunsell, 2009; Mitchell et al., 2009).

We grouped pairs of neurons based on the distance of the electrodes from which they were recorded and computed the mean and SEM of $r_{\text {sc }}$ within each distance bin (Fig. 2A). For both animals, $r_{\mathrm{sc}}$ decreased with increasing separation between the electrodes $(r=-0.120, p<0.0001$; Fig. $2 A$ shows binned data for illustration, but the correlation test was performed on the 3240 total pairs).

A number of previous studies have reported that pairs of neurons with similar visual tuning exhibit the strongest spike count correlation, whereas pairs with dissimilar or opposite tuning are less correlated (Zohary et al., 1994; Lee et al., 1998; Bair et al., 2001; Kohn and Smith, 2005; Smith and Kohn, 2008). This was also true in our V4 data. We measured the tuning similarity of a pair of neurons by taking the Pearson's correlation of their tuning curves to the 12 grating stimuli $\left(r_{\text {signal }}\right)$. There was a significant positive correlation between $r_{\text {sc }}$ and $r_{\text {signal }}(r=0.147, p<0.0001$; 
A
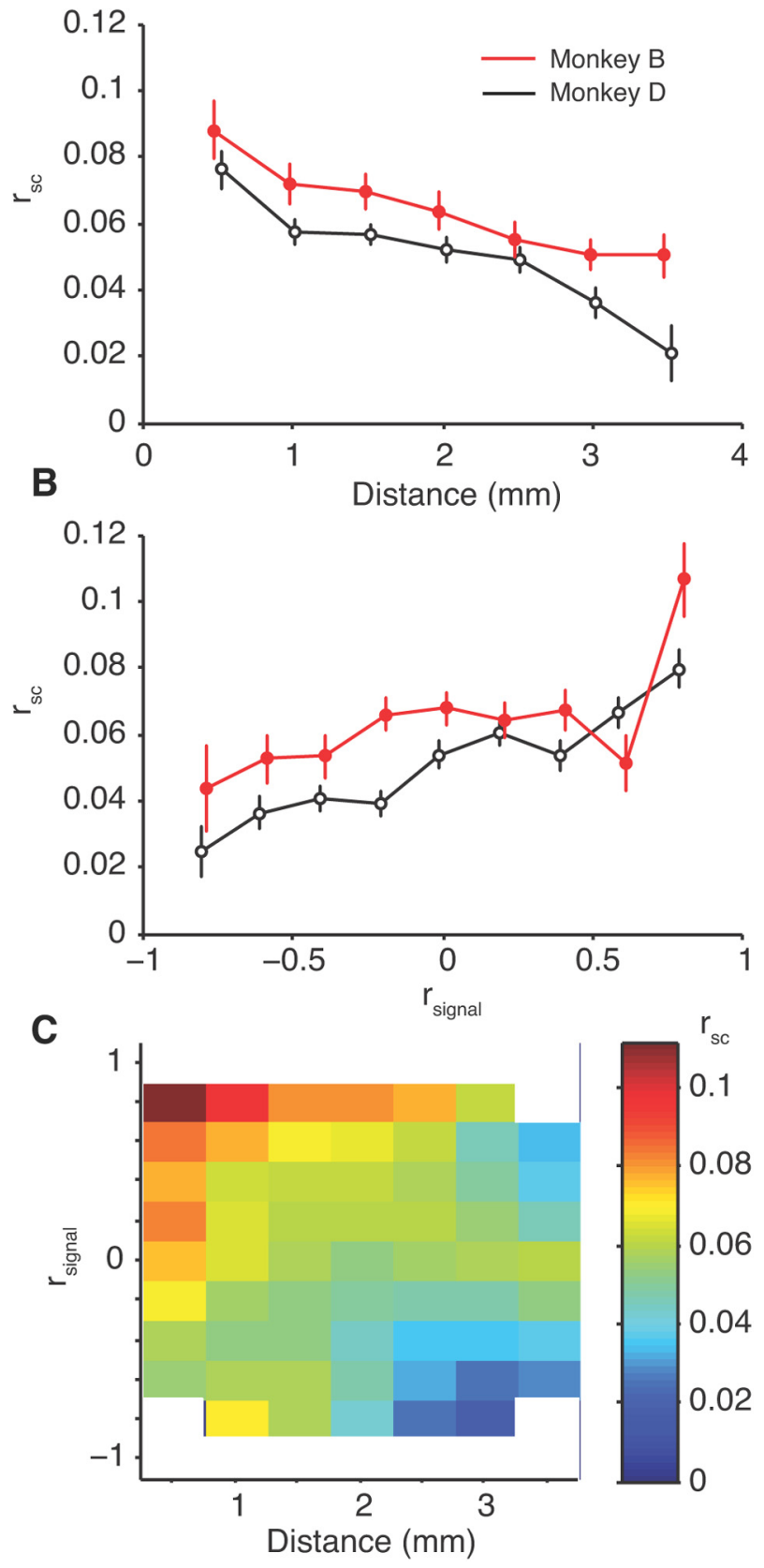

Figure 2. Correlated variability. $A$, Average $r_{\mathrm{sc}}$ for pairs of neurons as a function of distance. Neuronal pairs were grouped in $0.5 \mathrm{~mm}$ distance groups starting at $0.25 \mathrm{~mm}$. The red line represents data from Monkey $B$ and the black line represents data from Monkey D. B, Average $r_{s c}$ for pairs of neurons as a function of $r_{\text {signal }}$ or tuning similarity. $C$, Dependence of $r_{\mathrm{sc}}$ on both distance and tuning similarity for the pooled data from both monkeys. Bins containing $<10$ pairs are removed (indicated with white). The scale of the surface plot is indicated by the bar at the right of the panel. The data in this and all subsequent surface plots were smoothed with a two-dimensional Gaussian (SD of 1 bin) for display purposes only.

$n=3240$ pairs), indicating that neurons with similar orientation tuning tended to have more strongly correlated trial-to-trial variability (Fig. 2B).

In the two comparisons described above and in all other conditions we compared, the results were substantively similar between the animals (exceptions are noted below). We therefore pooled the data from both animals for the remainder of the analyses. Figure $2 C$ summarizes the relationship between $r_{\text {sc }}, r_{\text {signal }}$, and distance for all of the data from both animals. Pairs of neurons with the largest $r_{\mathrm{sc}}$ (indicated with red) tended to be close together and show similar orientation tuning (Fig. 2C, upper left of plot). Neurons with the smallest $r_{\text {sc }}$ were physically distant and had dissimilar tuning (Fig. 2C, blue regions, lower right of plot).

Variations among neuronal responses based on the stimulus history could conceivably affect measures of correlation. To determine whether such effects were present in our data, we ranked the stimuli from best to worst for each neuron. We then computed $z$-scored rates for each trial from each neuron and then found the average $z$-scored rate after each stimulus, sorted by rank. The goal was to measure whether the presentation of a good or bad stimulus tended to have a measurable effect on the subsequent stimulus. Responses were not significantly different between the stimuli that followed the best stimulus compared with the stimuli that followed the worst stimulus $(p=0.904)$ and there was no trend for the responses to vary as a function of preceding stimulus rank. We conclude that stimulus history does not play a significant role in influencing the correlation measures we report here.

In area $\mathrm{V} 1$, where there is a columnar structure for orientation, nearby neurons have similar orientation tuning. This kind of trend might manifest in our data as a negative correlation between $r_{\text {signal }}$ and distance. In our previous study in V1 (Smith and Kohn, 2008), we found no such interaction in the full dataset $(r=0.000, p=0.947)$, although one of the seven implants did show a significant negative correlation. Here, in V4, we found similarly inconsistent results between implants - there was a negative correlation between $r_{\text {signal }}$ and distance in one animal $(r=$ $-0.125, p<0.0001)$, but not in the other $(r=-0.034, p=0.193)$. We conclude that the presence or absence of such a trend in a particular array implant depends on the spacing of the electrodes in the array relative to the features of the cortical environment where the implant is performed, such as columnar structure, lateral connections, and interareal projection patterns.

\section{Time scales of correlated variability}

Correlated trial-to-trial variability can arise from network interactions on multiple time scales. Our measurement of $r_{\mathrm{sc}}$ was made over the full $1000 \mathrm{~ms}$ duration of the stimulus. To extend this analysis, we used another metric, $r_{\mathrm{ccg}}$, calculated as the integral of the CCG normalized by the area under the autocorrelograms of the neurons in the pair. By changing the temporal window of integration, $r_{\mathrm{ccg}}$ is useful for assessing correlation arising from precise temporal coordination (when the window is small) all the way up to long time scale interactions (when the window is large). When the window of integration is set to the full stimulus duration, $r_{\mathrm{ccg}}$ is mathematically equivalent to $r_{\mathrm{sc}}$ (Bair et al., 2001).

Figure $3 A$ shows the value of $r_{\text {ccg }}$ computed over a range of temporal integration windows for neuronal pairs with a range of separations. Cross-sections of this surface (Fig. $3 C$ ) in four distance groups $\left(0-4.0 \mathrm{~mm}\right.$ in $1.0 \mathrm{~mm}$ steps) reveal that $r_{\mathrm{ccg}}$ increases as a function of the size of the temporal integration window, reaching a peak at the full stimulus duration $(1000 \mathrm{~ms})$. The value of $r_{\text {ccg }}$ at this point (the rightmost edge) is equivalent to $r_{\mathrm{sc}}$. The time scale of correlation had a similar dependence on tuning similarity, as shown in Figure $3 B, D$.

\section{Synchrony}

Synchrony is a measure of pairwise correlations on a millisecond time scale and is generally measured by a shuffle-corrected CCG. As described above, the value of $r_{\mathrm{sc}}$ is related to the area under the 

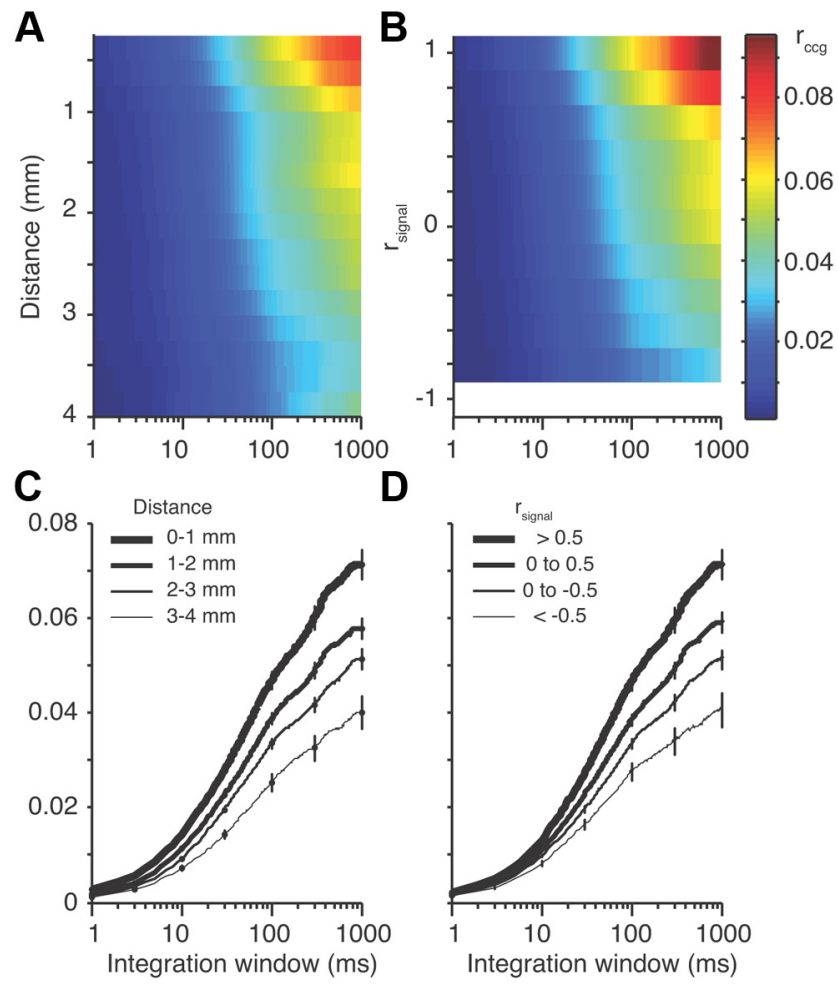

D

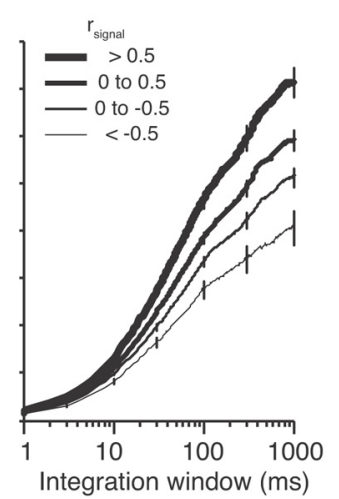

Figure 3. Effect of distance and tuning similarity on the time scale of correlation. $\boldsymbol{A}$, Surface plot showing the value of $r_{\text {ccg }}$ as a function of the distance between electrodes for a range of integration windows (1 ms up to $1 \mathrm{~s}$ ). All data in this figure are pooled from both monkeys. $\boldsymbol{B}$, Analogous surface plot of the value of $r_{c c g}$, this time as a function of the tuning similarity $\left(r_{\text {signal }}\right)$. The blank white region at the bottom indicates that no neuronal pairs had a $r_{\text {signal }}$ value between -0.9 and -1.0 . The scale bar for $\boldsymbol{A}$ and $\boldsymbol{B}$ is shown at the right. $\boldsymbol{C}, \boldsymbol{D}$, Line plots of $r_{\text {ccg }}$ as a function of integration window taken for several cuts from the surfaces in $\boldsymbol{A}$ and $\boldsymbol{B}$.

full shuffle-corrected CCG. In Figure $4 A$, we show this shufflecorrected CCG (black lines) calculated for neuronal pairs grouped by electrode separation in $0.5 \mathrm{~mm}$ increments. The effect described above, a decrease in $r_{\mathrm{sc}}$ as a function of distance, is clearly visible in the decrease in the area under the CCG as the pairs of neurons were increasingly farther apart. Inspection of the overall shape of the CCG revealed that there were interactions on at least two time scales, a sharp peak on the order of tens of milliseconds riding on top of a broader hill spanning hundreds of milliseconds. This was similar to our previous findings in V1, which also demonstrated two dominant time scales of neuronal interactions (Smith and Kohn, 2008).

We used a jitter-correction method to isolate neuronal interactions on fast time scales from correlated changes in trial-to-trial variability on a long time scale (see Materials and Methods and Smith and Kohn, 2008). In our prior study of V1 (Smith and Kohn, 2008), we found that a jitter window of $50 \mathrm{~ms}$ abolished correlation on a time scale longer than the window size and effectively isolated the synchronous interactions among neurons from the slower time scale interactions. Here we analyzed our V4 data in the same way, with jitter-corrected CCGs superimposed on the corresponding shuffle-corrected CCGs (Fig. 4A, gray lines, and Fig. $4 B$, showing the same jitter-corrected data magnified). It is clear that the slow interactions are removed; the jittercorrected CCG shows only a peak on brief time scales $(\sim 5-10 \mathrm{~ms}$ half-width).

To quantify these pairwise synchrony effects, we calculated the area under the jitter-corrected CCG peak for each pair of neurons, integrating from -10 to $+10 \mathrm{~ms}$ around the zero time

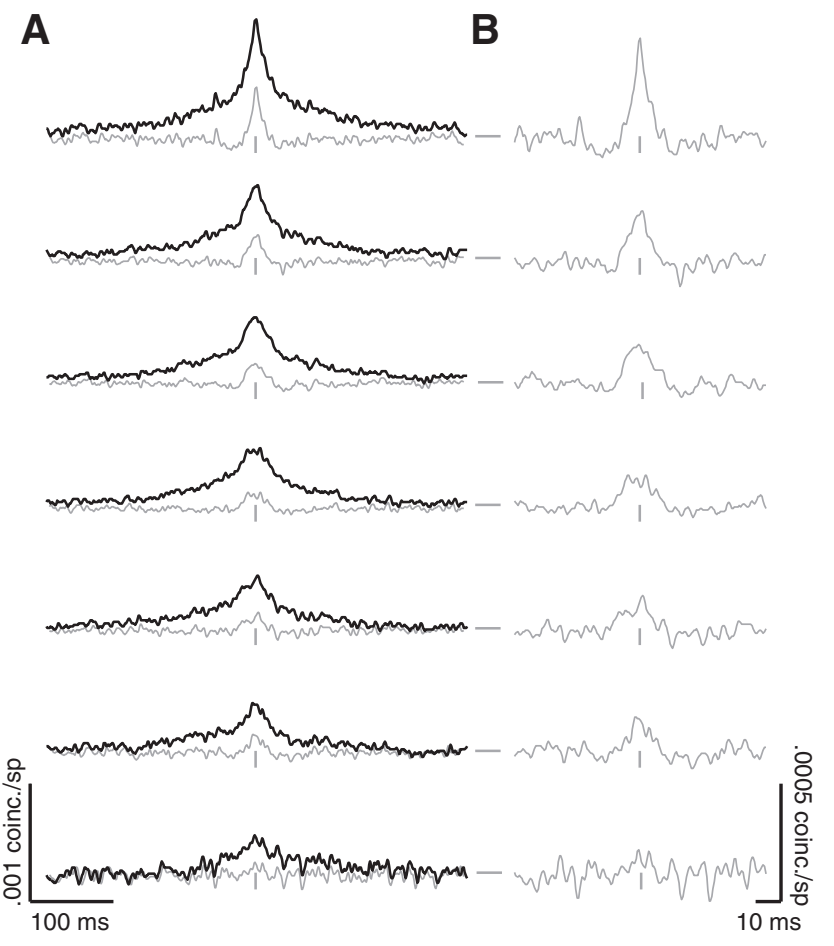

Figure 4. Synchrony. $\boldsymbol{A}$, Shuffle-corrected CCGs (black line) and jitter-corrected CCGs (gray lines, with a jitter window of $50 \mathrm{~ms}$ ) for pairs of neurons in V4. Nearby neurons are shown at the top and distant pairs at the bottom in $0.5 \mathrm{~mm}$ bins. The vertical gray ticks represent zero time lag and the horizontal gray ticks represent 0 coinc./sp. Vertical and horizontal scale bars are shown at the bottom left. $\boldsymbol{B}$, The same jitter-corrected CCGs as in $\boldsymbol{A}$, but with an expanded vertical and horizontal scale (indicated with the scale bars at the bottom right).

lag bin, and averaged this value for sets of neurons within each bin of pair separation distances (Fig. 5A). Similar to our result for correlated variability $\left(r_{\mathrm{sc}}\right)$, we found that synchrony was stronger among nearby neurons and decreased with distance $(r=-0.147$, $p<0.0001)$. We also grouped neurons based on their tuning similarity $\left(r_{\text {signal }}\right.$, Fig. $\left.5 B\right)$. Neurons with similar tuning tended to fire more synchronously $(r=0.051, p=0.004)$. Although highly significant, this effect was weaker than the distance dependence. Unlike the rest of the findings that we report, this effect was present in only one of the two monkeys (Monkey D, $r=0.111$, $p<0.0001$; Monkey B, $r=0.013, p=0.63)$.

We further analyzed pairs of neurons for synchronous interaction considering distance and tuning similarity simultaneously (Fig. 5C). We found a general trend for nearby neurons with similar tuning preferences to have more synchrony, which is consistent with our findings in V1 (Smith and Kohn, 2008). However, in V1, we saw synchrony drop to values indistinguishable from zero for pairs of neurons $2 \mathrm{~mm}$ or more apart, whereas synchrony in V4 remained above zero at that distance. Therefore, the falloff of synchrony with distance was steeper in V1 than in V4. We know, however, that the spatial scale of correlation can depend on the time scale of analysis (Smith and Kohn, 2008), so it seemed possible that this could account for the apparent lack of complete falloff in V4. To test this possibility, we performed an additional analysis designed to compare synchronous activity across different time scales. We computed jitter-corrected CCGs with a range of window sizes $(10,20,50,100 \mathrm{~ms})$. In each case, we computed the synchrony as the area under the CCG peak within half the window size of the zero time lag bin $(5,10,25,50 \mathrm{~ms})$. We then normalized the average synchrony values to a peak value of 1. Figure $6 A$ shows normalized synchrony as a function of dis- 


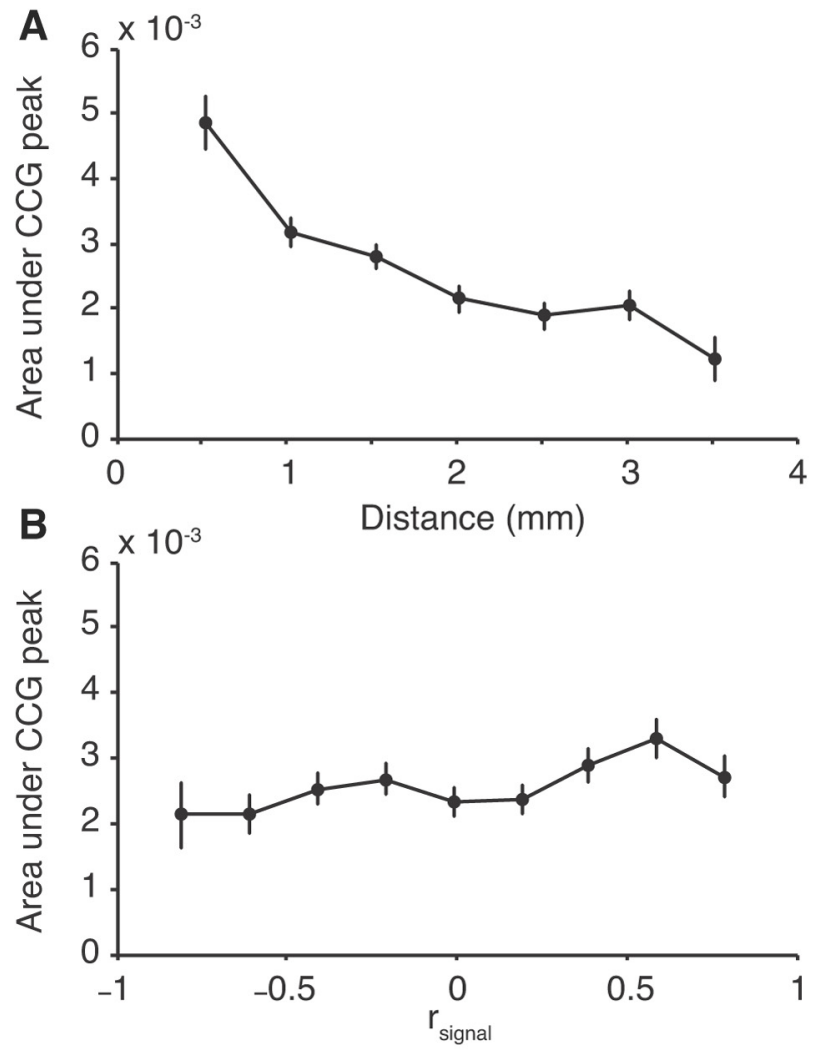

C

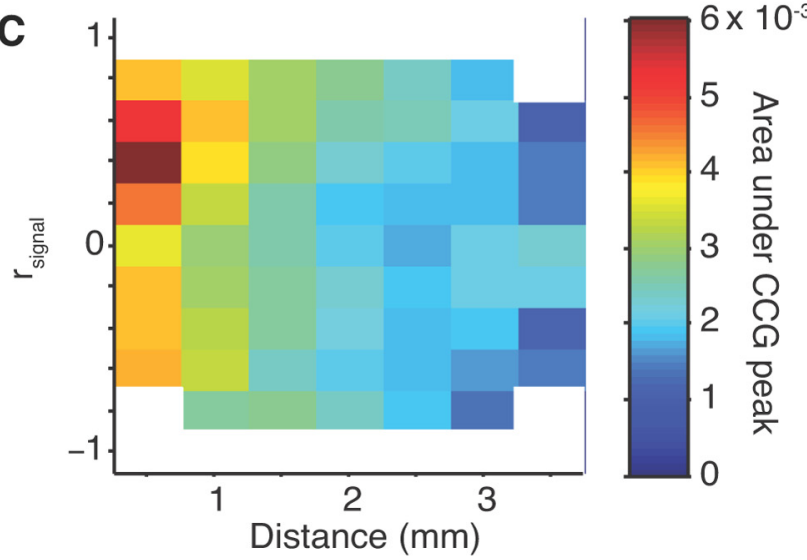

Figure 5. Influence of distance and tuning similarity on synchrony. $\boldsymbol{A}$, Synchrony, as measured by the average area under the $C(G$ within $10 \mathrm{~ms}$ of the zero time lag bin as a function of the distance between neurons. $\boldsymbol{B}$, Synchrony as a function of tuning similarity $\left(r_{\text {signal }}\right)$. C, Dependence of synchrony on both distance and tuning similarity shown as a surface plot. Bins containing $<10$ pairs are removed (indicated with white). The bar at the right of the panel indicates the scale.

tance plotted for these various jitter windows (solid gray and black lines), for a shuffle-corrected CCG (dashed black line with area calculated under the entire CCG), and for the V1 data from Smith and Kohn (2008) that used a $50 \mathrm{~ms}$ jitter window (red line with open symbols). Synchrony in V4 on a very fine timescale (10 ms window, i.e., pairwise spiking of two neurons with synchrony $\leq 5 \mathrm{~ms}$, thick gray line) had a distance dependence that closely matched that in $\mathrm{V} 1$ at a much longer time scale $(50 \mathrm{~ms})$. For longer time scales in V4 (50-100 ms, thin black and dark gray lines), the distance dependence was similar to that for the shufflecorrected CCG (and thus to $r_{\mathrm{sc}}$ ). This implies that, in V4, only correlated spiking with a very high temporal precision $(\leq 5 \mathrm{~ms})$ has a tight spatial extent, whereas even moderately precise align-
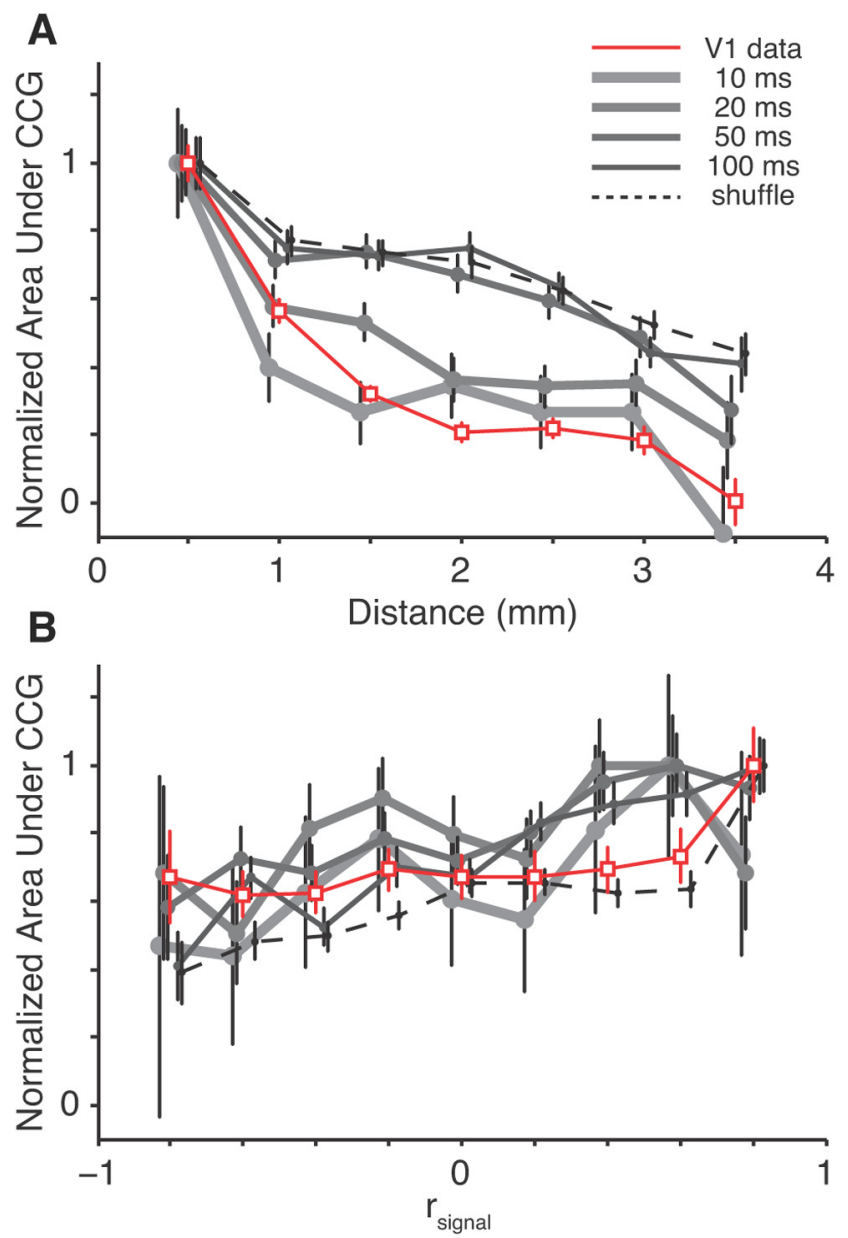

Figure 6. Measuring the time scale of synchrony. $A$, Normalized synchrony (adjusted to a peak of 1 ) as a function of distance between neurons. The dashed line represents the shufflecorrected CCGs, the solid gray lines represent jitter-corrected CCGs of various temporal windows, and the solid red line represents V1 data from Smith and Kohn (2008) using a jitter window of $50 \mathrm{~ms}$. In the $\mathrm{V} 4$ lines, the area under the peak is measured for plus and minus one half of the jitter window on either side of the zero time lag bin. For the shuffle-corrected $C(G$, area was measured under the entire $C \subset G( \pm 1 \mathrm{~s})$. $\boldsymbol{B}$, Normalized synchrony, with the same conventions as in $\boldsymbol{A}$, plotted as a function of tuning similarity $\left(r_{\text {signal }}\right)$.

ment (50 ms or more) has a relatively broad spatial distribution. This is not to say that $>50 \mathrm{~ms}$ synchrony is unlimited in space across V4; its limit was simply beyond the spatial extent that our arrays could test.

We also computed synchrony as a function of tuning similarity using similar analyses (Fig. 6B). Here, the V1 data (red line) seem to fall within the range of the V4 data. For both V4 and V1, on long and short time scales, synchrony is only weakly correlated with $r_{\text {signal. }}$.

Although the average CCG metrics in V4 agreed well with V1, we did notice that among individual pairs of V4 neurons, CCGs with an obvious peak near zero were substantially less frequent than we had observed previously in V1. Employing the same metric we used previously in V1 (Smith and Kohn, 2008), we determined for each pair whether the jitter-corrected CCG (50 ms window) had a significant peak. A peak was deemed significant if it had a bin within $10 \mathrm{~ms}$ of zero time lag that exceeded 5 SDs of the noise level (averaged values from -250 to -200 and from 200 to $250 \mathrm{~ms}$ ). For nearby pairs ( $<0.75 \mathrm{~mm}$ ), only $3.6 \%$ (23 of 645) fulfilled this criterion in V4, whereas in V1, 13\% of pairs at this distance had significant peaks (Smith and Kohn, 
2008). As in V1, we did observe that in V4, the number of significant peaks decreased with distance ( $1.3 \%$ of peaks $>0.75 \mathrm{~mm}$ ). To quantify this observation, we calculated the percentage of pairs that had significant peaks at each distance in $0.25 \mathrm{~mm}$ bins starting from the nearest neurons and proceeding to the most distant pairs we recorded. We found that the percentage of significant pairs decreased sharply with increasing separation (Spearman's $\rho=-0.87, p<0.0001$ ). Therefore, although we have noted some differences between synchrony in V4 and in V1, the overall trend was for similar characteristics in the two areas, such as dependence on distance and tuning similarity.

\section{Correlation of spontaneous activity}

Spontaneous cortical activity tends to be more correlated than evoked responses (Kohn and Smith, 2005; Smith and Kohn, 2008). This effect has been reported primarily in anesthetized animals and has not been explored thoroughly in the awake state. Correlation decreases among V4 neurons when attention is directed at their receptive fields (Cohen and Maunsell, 2009; Mitchell et al., 2009) and among neurons in rat barrel cortex during active whisking (Poulet and Petersen, 2008). Therefore, there is some reason to believe that the difference between spontaneous and evoked activity is a general one that is present in anesthetized and awake animals and across sensory domains. We tested this in our experiment by recording data while the animals fixated with a blank screen present. In the two monkeys, we recorded 1122 pairs of neurons in which both had a sufficient firing rate $(2 \mathrm{sp} / \mathrm{s})$ to compute $r_{\mathrm{sc}}$. The mean $r_{\mathrm{sc}}$ in that group was $r=$ $0.110 \pm 0.003$, greater than the evoked $r_{\mathrm{sc}}$ value computed above $(p<0.0001)$. The value of $r_{\text {sc }}$ in the spontaneous (dashed line) and evoked (solid line) conditions is shown in Figure $7 A$, binned by the distance between neurons. At all of the distances, the spontaneous $r_{\mathrm{sc}}$ is greater than the evoked $r_{\mathrm{sc}}$ by a factor of 1.9 on average. This ratio is very close to that observed in V1 (1.7; Smith and Kohn, 2008). The value of $r_{\mathrm{sc}}$ was also correlated between the spontaneous and evoked conditions $(r=0.215, p<0.0001)$, but the $r_{\mathrm{sc}}$ during the spontaneous state was not predicted by the value of $r_{\text {signal }}$ in the evoked data for the same pairs $(r=0.035$, $p=0.289$ ).

We also examined whether this difference between spontaneous and evoked $r_{\text {sc }}$ would hold at all time scales. We computed the $r_{\text {ccg }}$ for both spontaneous (dashed line) and evoked (solid line) conditions shown in Figure $7 B$. We found that at all time scales, spontaneous activity was more correlated than evoked activity. This is consistent with our observations of $r_{\mathrm{sc}}$ on a long time scale and with a previous report in V1 Kohn and Smith, 2005).

\section{Interactions on long time scales}

Correlation can also arise from slow processes, such as motivation or arousal, that act over a time scale of greater than one trial (on the order of several seconds). One way to look for such effects is to compute the trial cross-covariance (TCC) of the spike counts (Bair et al., 2001), essentially the $r_{\text {sc }}$ computed with various trial offsets for each pair. At a trial lag of zero, the TCC is equal to the value of $r_{\mathrm{sc}}$. For a given trial lag, a nonzero value indicates that the spike count from one neuron in the pair is correlated with the spike count of the other neuron several trials later. Because our trials consisted of $1 \mathrm{~s}$ of stimulus presentation, a 1-trial lag is at least $1 \mathrm{~s}$ plus the time required for the animal to make a saccade to the target and return to fixation. In practice, the median time between the start of one trial and the start of the next was $2.5 \mathrm{~s}$.
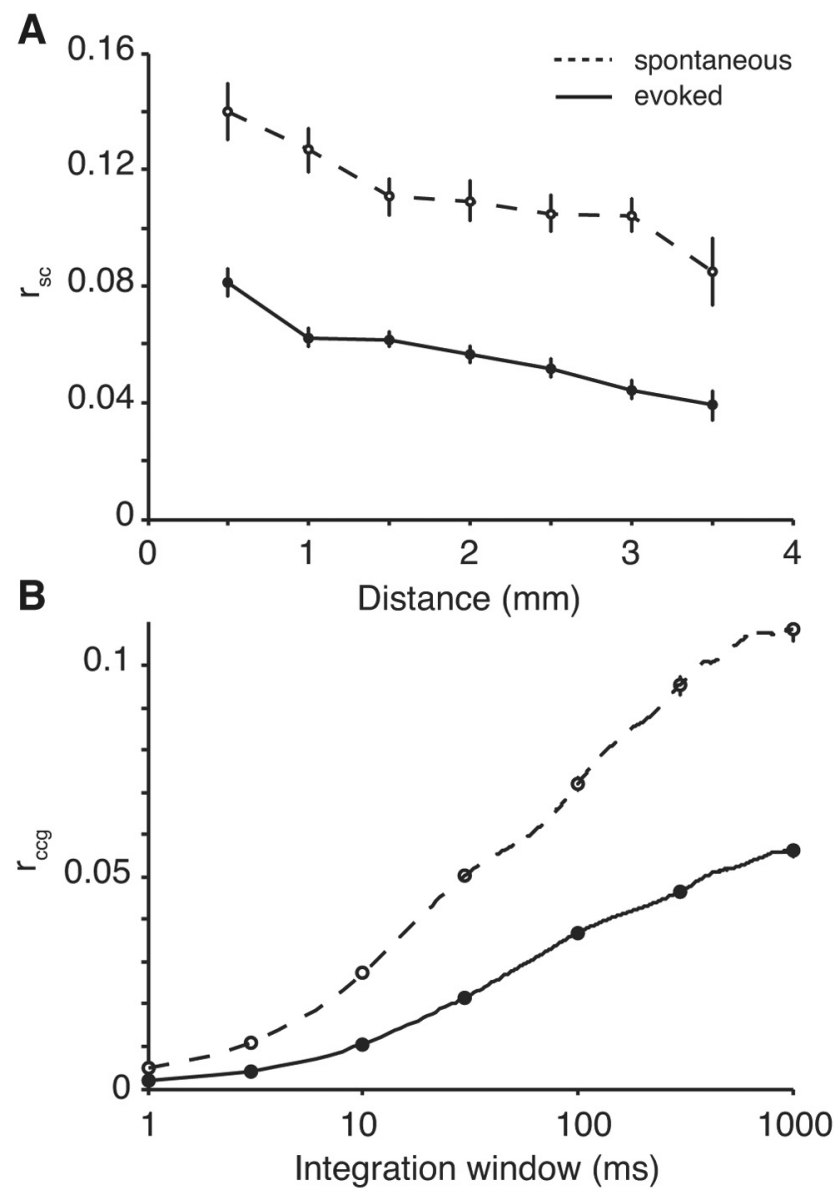

Figure 7. Differences between spontaneous and evoked activity. $\boldsymbol{A}$, Correlated variability $\left(r_{s c}\right)$ as a function of the distance between neurons for spontaneous (dashed line) and evoked (solid line) activity. $\boldsymbol{B}$, Time scale of correlation is shown here for the same neurons, with a plot of $r_{c c g}$ as a function of integration window.

The TCC values for our evoked (thin black line with SEM indicated by the shaded gray region around the line) and spontaneous (thin red line with light red shaded region) V4 data are shown in Figure 8. The value of the TCC was clearly greater than zero away from the central point, indicating that some long-timescale fluctuations were present in the data that affected $r_{\text {sc }}$. To assess these effects, we replaced the value at zero with the average of the adjacent values and smoothed the curves for evoked (Fig. 8, thick black line in inset panel) and spontaneous (thick red line) activity (following the procedure of Bair et al., 2001). The value of $r_{\mathrm{sc}}$ can be considered to be approximately the sum of a short term $\left(r_{\mathrm{st}}\right)$ and long term $\left(r_{\mathrm{lt}}\right)$ component. The value of $r_{\mathrm{lt}}$ is read from the smoothed curves at zero, and the value of $r_{\text {st }}$ can be approximated as the difference between $r_{\mathrm{sc}}$ and $r_{1 \mathrm{t}}$. This analysis complements the $r_{\text {ccg }}$ metric above in that it provides a view of the constituents of $r_{\mathrm{sc}}$, this time on long time scales.

Across all pairs of neurons, the value of $r_{1 \mathrm{t}}$ in evoked (0.016 \pm $0.0005)$ and spontaneous $(0.010 \pm 0.0006)$ activity was significantly greater than zero $(p<0.0001)$ and was $\sim 25 \%$ of the magnitude of $r_{\mathrm{sc}}$ at lag $=0$ in the evoked case (and $8 \%$ in the spontaneous case), indicating the presence of some amount of correlation that fluctuates at a time scale of many seconds to minutes. The value of $r_{\mathrm{lt}}$ was larger $(p<0.0001)$ in the evoked than spontaneous case, which indicates that part of this longterm fluctuation is related to the presence of a visual stimulus. The tuning similarity of a pair of neurons was unrelated to $r_{1 \mathrm{t}}(r=$ 


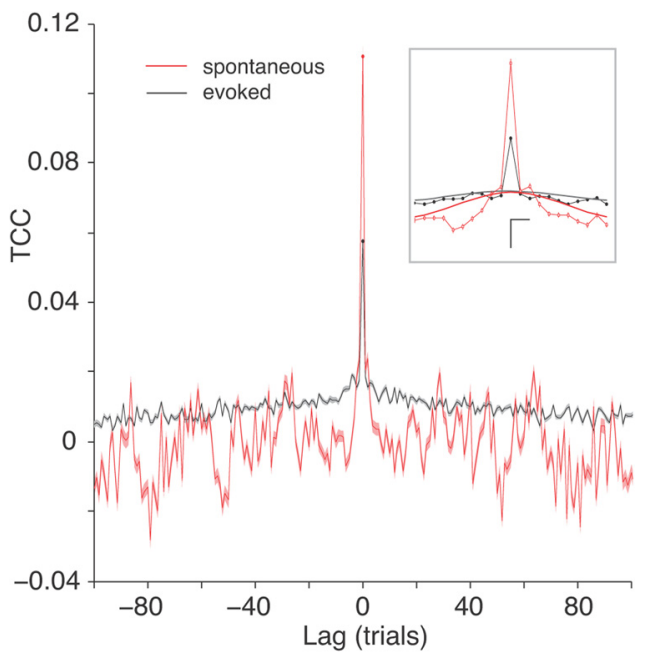

Figure 8. Long-time-scale correlation. TCC is shown for spontaneous (red line) and evoked (black line) activity. The SEM around each data point is shown as a shaded region (red or light gray) around the TCC. The TCC shows the amount of correlated variability as a function of the trial lag between the responses of the neurons. For example, at a lag of one trial, the responses of one neuron are correlated with the responses of another neuron collected during the subsequent stimulus. The value at a lag of zero trials is the same as $r_{\text {sc }}$ The inset shows an enlarged view of the data near a trial lag of zero. The thick gray and red lines represent fits to the TCC data with the zero-lag point excluded (see Materials and Methods) for evoked and spontaneous data, respectively. The vertical scale bar represents a TCC value of 0.02 and the horizontal scale bar represents a lag of 2 trials. The intersection of the two scale bars indicates the origin.

$-0.003, p=0.87)$, but there was a small but significant correlation between $r_{1 \mathrm{t}}$ and the distance between neurons $(r=0.036, p=$ $0.039)$. The value of $r_{\mathrm{lt}}$ was also correlated with $r_{\mathrm{sc}}$ in both the evoked $(r=0.516, p<0.0001)$ and spontaneous $(r=0.210, p<$ $0.0001)$ conditions.

The value of the TCC decreased rapidly as the trial lag increased. Given that the length of time between trials is not constant because of fluctuations in behavioral performance, it is difficult to establish the true time course of the slow fluctuations underlying $r_{1 t}$. In the evoked response, there appeared to be two processes giving rise to long-time-scale correlation. The value of the TCC was highest within $\sim 10$ trials of zero lag, after which it dropped to approximately half the magnitude and remained above zero until trial lags of 50-100. In our two animals, the median time between successfully completed trials was $2.5 \mathrm{~s}$. This would indicate that processes of $\sim 25 \mathrm{~s}$ and $\sim 2-5 \mathrm{~min}$, respectively, contributed to the slow fluctuations we measured in the evoked response.

\section{Effects of rate on correlation}

Firing rate is known to influence the magnitude of neuronal correlation-neuronal pairs that fire more are likely to have larger values for $r_{\mathrm{sc}}$ and synchrony (de la Rocha et al., 2007; Smith and Kohn, 2008). In our population of 113 neurons, the average firing rate was $5.5 \pm 0.52 \mathrm{sp} / \mathrm{s}$ in response to the grating stimuli (averaged across all orientations). In all of the pairs recorded among these neurons, there was a significant correlation between the geometric mean firing rate of the pair and the $r_{\mathrm{sc}}(r=0.32, p<$ $0.0001, n=3240)$. This was also true for synchrony as calculated for Figure 4 and Figure $5(r=0.32, p<0.0001, n=3240)$. There was, however, no relationship between firing rate and distance $(r=-0.01, p=0.44)$, indicating that changes in firing rate alone cannot explain the results presented here.

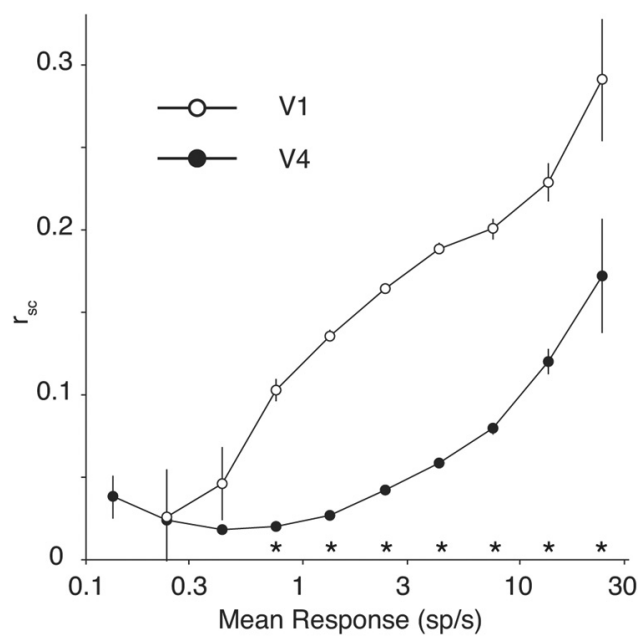

Figure 9. Correlation and rate. The spike count correlation $\left(r_{s c}\right)$ is plotted here as a function of the mean firing rate of the neuronal pair for V1 (open circles) and V4 (filled circles). The V1 data are taken from Smith and Kohn (2008) and were recorded in anesthetized macaque monkey. Asterisks indicate a significant difference ( $p<0.05$, one-tailed $t$ test) between V4 and V1 For rate-matched groups of neurons, $r_{s c}$ is larger in V1 than V4 across a range of firing rates from $0.75 \mathrm{sp} / \mathrm{s}$ to the highest rates we recorded. Note that there were only five total pairs of V1 neurons that comprised the data for the leftmost two open circles.

The magnitude of spike count correlation we observed in V4 (0.058) was less than in our previous report in V1 (0.176; Smith and Kohn, 2008). Given the influence of rate on correlation, a difference in firing rates between V1 and V4 could explain this result. To determine if this was the case, we took the geometric mean firing rate for a pair of neurons to each orientation of the stimulus and then averaged across the orientations to produce a single measure of the responsiveness of each pair. We then logtransformed this value to normalize the firing rate distributions and grouped pairs of neurons with similar mean rates. The value of $r_{\mathrm{sc}}$ is shown for V1 (open circles) and V4 (filled circles) as a function of mean rate in Figure 9. The average $r_{\mathrm{sc}}$ in V1 was significantly higher $(p<0.05$, one-tailed $t$ test) than V4 for pairs of neurons in every group $>0.75 \mathrm{sp} / \mathrm{s}$ (only 5 pairs of V1 neurons were below this mean rate). Therefore, the lower magnitude of $r_{\mathrm{sc}}$ in V4 compared with V1 cannot be explained by differences in firing rate.

\section{Discussion}

We found that correlations in V4 depend on both interneuronal distance and tuning similarity. Across time scales, correlations were greater for spontaneous than stimulus-evoked activity, approximately by a factor of two. The results were qualitatively similar to those we found in V1, although quantitatively were more modest.

\section{Correlated trial-to-trial variability}

Few studies have attempted to measure correlated variability thoroughly across neurons. Two previous investigations in V4 (Cohen and Maunsell, 2009; Mitchell et al., 2009) used an attentional task. Cohen and Maunsell (2009) reported that correlated variability was higher for V4 pairs of neurons with similar orientation tuning, but was not dependent on distance. The former result agrees with our data, but the latter does not. Their study used a $6 \times 8$ Utah array, with a maximum electrode separation approximately two-thirds as large as our $10 \times 10$ array. Given the relatively slow decrease in correlated variability with distance, the decrease may have been less noticeable in their data. Alterna- 
tively, the falloff of cortical variability with distance may depend on factors that vary with eccentricity; their arrays were implanted more dorsally than ours, leading to more eccentric receptive fields. There is some evidence that central and peripheral V4 have different interareal connection patterns (Ungerleider et al., 2008). Cohen and Maunsell (2009) did not find significant correlated variability between neurons in opposite hemispheres, indicating that for very large distances, neurons in V4 are effectively independent.

Most studies of correlated variability report similar numbers across a wide variety of stimuli and recording preparations (for review, see Cohen and Kohn, 2011), but one recent study was an exception. Ecker et al. (2010) reported a mean value of $r_{\text {sc }}$ in primate $\mathrm{V} 1$ of 0.01 , significantly greater than but very close to zero. They suggested that larger values of $r_{\mathrm{sc}}$ from previous studies were due to errors in spike sorting or uncontrolled cognitive factors. We did not include pairs of neurons recorded from the same electrode and our results were robust to changes in criteria for included units (adjusting the SNR threshold), indicating that spike-sorting errors were not the source of our larger $r_{\mathrm{sc}}$. Our animals performed a basic fixation task during which attention might have influenced V4 activity; in principle, fluctuations in attention from trial to trial could lead to an increase in observed $r_{\text {sc }}$. However, it is unlikely that such fluctuations explain our $r_{\mathrm{sc}}$ magnitude, given its similarity to that found in other studies of V4 (Cohen and Maunsell, 2009; Mitchell et al., 2009) and V1 (Smith and Kohn, 2008). Finally, unless downstream neurons have access to the source of such fluctuations and can factor them out, they would appear as "noise" to those neurons.

\section{Synchrony}

In the visual system, synchrony has long been proposed as a mechanism for binding object representations (Gray, 1999) and for coordinating information transmission between cortical regions (Fries, 2009). More recently, synchrony has been proposed as a substrate for attention effects in V4. Most studies have focused on gamma oscillations (typically $30-80 \mathrm{~Hz}$ ) as the measure of synchrony (Fries et al., 2001; Bichot et al., 2005; Taylor et al., 2005; Rotermund et al., 2009), with less investigation of synchronous spiking activity. Cohen and Maunsell (2009) found only chance levels using a shuffle-corrected CCG. However, their 200 $\mathrm{ms}$ analysis window may have made detection difficult due to small numbers of spikes. We also found only a small percentage of pairs of V4 neurons with significant CCG peaks, but by averaging the noisy signals present in individual CCGs, we were able to measure the structure of synchrony. Our study thus represents the first systematic investigation of spiking synchrony in area V4. We found that the presence of synchronous spiking activity, at very fine temporal scales ( $5 \mathrm{~ms}$ ), was highly dependent on distance: it was present almost exclusively among neurons separated by $<2 \mathrm{~mm}$. Synchrony also tended to be stronger among neurons with similar tuning.

\section{Comparison between V4 and V1}

We concluded that V4 differs from V1 in two distinct ways: it had weaker correlated variability and less temporally precise synchrony. The spike count correlation we observed in V4 (0.06) was consistent with two recent studies in V4 (0.04 by Cohen and Maunsell, 2009; 0.05 by Mitchell et al., 2009), but markedly less than that found in V1 recordings (range, 0.1-0.25 in Kohn and Smith, 2005; Gutnisky and Dragoi, 2008; Smith and Kohn, 2008; Poort and Roelfsema, 2009; Samonds et al., 2009; Rasch et al., 2011). Our previous study in V1 (Smith and Kohn, 2008) used methods and analyses most closely matching those used here and thus provides the best direct comparison. The present study differed from that one in three notable ways. First, Smith and Kohn (2008) used opiate-anesthetized animals. Correlation values are similar, however, among V1 reports that used anesthetized (Kohn and Smith, 2005; Smith and Kohn, 2008) and awake (Gutnisky and Dragoi, 2008; Poort and Roelfsema, 2009; Samonds et al., 2009; Rasch et al., 2011) preparations, implying that effects of opiate anesthesia on $r_{\text {sc }}$ are negligible. Second, our V4 recordings were slightly deeper than our V1 recordings (1.0 vs $0.6 \mathrm{~mm}$ array insertion depths). Therefore, we were likely closer to the middle layers in V4. In V1, correlations depend on cortical layer, with a minimum value found in the middle layers (Hansen et al., 2012; Smith et al., 2013). However, in V2, the magnitude of correlation is relatively constant across layers (Smith et al., 2013), suggesting that a strong dependence of correlation on cortical layer may be unique to V1 (and perhaps other primary sensory regions). Laminar trends alone are thus an unlikely explanation for decreased correlations found in V4 vs V1. Finally, in our awake animals, it is possible that small eye movements during stimulus presentation introduced spurious correlations. For neurons with similar receptive fields, microsaccades could tend to bring the two receptive fields jointly into slightly better or worse alignment with the visual stimulus, leading to correlated modulation in the two neurons' firing rates from trial to trial. Because of an error in eye position data collection, we could not apply offline analysis to rule out this scenario definitively. However, microsaccade-related effects were unlikely for three reasons: (1) we found that $r_{\mathrm{sc}}$ was higher in spontaneous activity than evoked and microsaccades should have led to higher correlations in evoked activity as receptive fields were jittered over a visual stimulus; (2) the relationships between correlation and other factors (e.g., distance and firing rate) in V4 were remarkably similar to the relationships found in V1, for which data were collected under anesthesia and paralysis such that eye movements were absent and could not have affected correlations; and (3) another study in area V4 (Mitchell et al., 2009) reported a similar level of neuronal correlation as we observed and found that fixational eye movements had no appreciable effects on correlated activity. We conclude that the correlation differences we observed between V4 and V1 were not artifactual.

Synchrony in V4 neurons was somewhat less temporally precise than in V1, with a smaller proportion of V4 pairs having significant peaks, and yet the average CCG peak had a similar height. This may have in part been due to fewer trials in our V4 data and added noise from behavioral variability. In V4, synchrony fell off rapidly as the distance between neurons increased, but this was true only when isolating precise spiking (within $5 \mathrm{~ms}$ of the peak). In $\mathrm{V} 1$, the distinction between fast and slow time scales of correlation was made more easily. There are few data available on the precise time scales of correlation across visual cortex, and comparisons with other studies are difficult because analysis methods vary widely for computing synchrony. The data that are available show synchrony that is generally comparable to that observed here (Bair et al., 2001; Thiele and Stoner, 2003). Synchrony is also known to depend on stimulus features such as orientation (Gray and Viana Di Prisco, 1997; Friedman-Hill et al., 2000; Frien et al., 2000; Kohn and Smith, 2005) and spatial integrity (Zhou et al., 2008), leaving open the possibility that the differences we found between V4 and V1 might be due in part to the relative effectiveness of the grating stimuli at driving the neurons in each area.

The differences we observed between V4 and V1 are likely to reflect the local cortical architecture of the areas. Horizontal con- 
nections in V4 follow a similar profile to V1, but with somewhat larger patches with greater spacing (Yoshioka et al., 1992). V4 also appears to have a different pattern of connections centrally than peripherally (Ungerleider et al., 2008) and a functional organization for color and form (Tanigawa et al., 2010). All of these differences could affect neuronal correlation. Finally, correlations in various cortical circuits might also differ due to mechanisms such as gamma oscillations produced by recurrent networks or biophysical properties of each area's neurons (Traub et al., 2001; Hasenstaub et al., 2005; Morita et al., 2008).

\section{Differences between spontaneous and evoked activity}

Previous studies have described extensive structure in spontaneous population activity using methods as varied as intracellular recordings (Okun and Lampl, 2008; Poulet and Petersen, 2008), field potentials (Fukushima et al., 2012), optical imaging (Tsodyks et al., 1999; Kenet et al., 2003), and MRI (Nir et al., 2008). A comprehensive study of single-neuron variability across cortical areas (Churchland et al., 2010) showed a decrease shortly after stimulus onset, consistent with the idea that the prestimulus (or "spontaneous") period activity has higher correlated variability. We found that correlation in spontaneous activity, when measured over any time scale, exceeded that of evoked activity. Over the length of the trial, the difference was approximately twofold, very close to the ratio reported in V1 in anesthetized macaques (Smith and Kohn, 2008). It is therefore reasonable to ask about the ecological relevance of spontaneous state measurements. The spontaneous state, in both anesthetized and alert animals, may be closely related to the "unattended" condition (Cohen and Maunsell, 2009; Mitchell et al., 2009). When a visual stimulus is presented, attention is exogenously drawn to that stimulus, reducing correlation. A cue that affects the allocation of attention can further modulate correlation, pushing it closer to the attended or unattended conditions.

\section{Conserved structure of neuronal correlation}

In two previous studies in V1 (Kohn and Smith, 2005; Smith and Kohn, 2008), we demonstrated that synchrony and correlated variability have different stimulus dependence and spatial extent and suggested that at least two distinct mechanisms contribute to correlation. Here, we extend those findings to V4, a stage beyond V1. Despite the differences discussed above, the overall trend was for both correlated variability and synchrony to follow similar rules in the two areas. It is likely, therefore, that neuronal correlation is primarily driven by circuits that are highly conserved across different regions of visual cortex, such as recurrent feedforward/feedback and horizontal intracortical connections.

\section{References}

Abbott LF, Dayan P (1999) The effect of correlated variability on the accuracy of a population code. Neural Comput 11:91-101. CrossRef Medline

Amarasingham A, Harrison MT, Hatsopoulos NG, Geman S (2012) Conditional modeling and the jitter method of spike resampling. J Neurophysiol 107:517-531. CrossRef Medline

Averbeck BB, Latham PE, Pouget A (2006) Neural correlations, population coding and computation. Nat Rev Neurosci 7:358-366. CrossRef Medline

Bair W, Zohary E, Newsome WT (2001) Correlated firing in macaque visual area MT: time scales and relationship to behavior. J Neurosci 21:16761697. Medline

Bichot NP, Rossi AF, Desimone R (2005) Parallel and serial neural mechanisms for visual search in macaque area V4. Science 308:529-534. CrossRef Medline

Brainard DH (1997) The Psychophysics Toolbox. Spat Vis 10:433-436. CrossRef Medline
Churchland MM, Yu BM, Cunningham JP, Sugrue LP, Cohen MR, Corrado GS, Newsome WT, Clark AM, Hosseini P, Scott BB, Bradley DC, Smith MA, Kohn A, Movshon JA, Armstrong KM, Moore T, Chang SW, Snyder LH, Lisberger SG, Priebe NJ, et al. (2010) Stimulus onset quenches neural variability: a widespread cortical phenomenon. Nat Neurosci 13:369378. CrossRef Medline

Cohen MR, Kohn A (2011) Measuring and interpreting neuronal correlations. Nat Neurosci 14:811-819. CrossRef Medline

Cohen MR, Maunsell JH (2009) Attention improves performance primarily by reducing interneuronal correlations. Nat Neurosci 12:1594-1600. CrossRef Medline

Cohen MR, Newsome WT (2009) Estimates of the contribution of single neurons to perception depend on timescale and noise correlation. J Neurosci 29:6635-6648. CrossRef Medline

Ecker AS, Berens P, Keliris GA, Bethge M, Logothetis NK, Tolias AS (2010) Decorrelated neuronal firing in cortical microcircuits. Science 327:584-587. CrossRef Medline

Friedman-Hill S, Maldonado PE, Gray CM (2000) Dynamics of striate cortical activity in the alert macaque: I. Incidence and stimulus-dependence of gamma-band neuronal oscillations. Cereb Cortex 10:1105-1116. CrossRef Medline

Frien A, Eckhorn R, Bauer R, Woelbern T, Gabriel A (2000) Fast oscillations display sharper orientation tuning than slower components of the same recordings in striate cortex of the awake monkey. Eur J Neurosci 12:14531465. CrossRef Medline

Fries P (2009) Neuronal gamma-band synchronization as a fundamental process in cortical computation. Annu Rev Neurosci 32:209-224. CrossRef Medline

Fries P, Reynolds JH, Rorie AE, Desimone R (2001) Modulation of oscillatory neuronal synchronization by selective visual attention. Science 291: 1560-1563. CrossRef Medline

Fukushima M, Saunders RC, Leopold DA, Mishkin M, Averbeck BB (2012) Spontaneous high-gamma band activity reflects functional organization of auditory cortex in the awake macaque. Neuron 74:899-910. CrossRef Medline

Gray CM (1999) The temporal correlation hypothesis of visual feature integration: still alive and well. Neuron 24:31-47, 111-125. CrossRef Medline

Gray CM, Viana Di Prisco G (1997) Stimulus-dependent neuronal oscillations and local synchronization in striate cortex of the alert cat. J Neurosci 17:3239-3253. Medline

Gutnisky DA, Dragoi V (2008) Adaptive coding of visual information in neural populations. Nature 452:220-224. CrossRef Medline

Hansen BJ, Chelaru MI, Dragoi V (2012) Correlated variability in laminar cortical circuits. Neuron 76:590-602. CrossRef Medline

Hasenstaub A, Shu Y, Haider B, Kraushaar U, Duque A, McCormick DA (2005) Inhibitory postsynaptic potentials carry synchronized frequency information in active cortical networks. Neuron 47:423-435. CrossRef Medline

Hays A, Richmond B, Optican L (1982) A UNIX-based multiple process system for real-time data acquisition and control. In, pp 1-10.

Huang X, Lisberger SG (2009) Noise correlations in cortical area MT and their potential impact on trial-by-trial variation in the direction and speed of smooth-pursuit eye movements. J Neurophysiol 101:3012-3030. CrossRef Medline

Judge SJ, Richmond BJ, Chu FC (1980) Implantation of magnetic search coils for measurement of eye position: an improved method. Vision Res 20:535-538. CrossRef Medline

Kelly RC, Smith MA, Samonds JM, Kohn A, Bonds AB, Movshon JA, Lee TS (2007) Comparison of recordings from microelectrode arrays and single electrodes in the visual cortex. J Neurosci 27:261-264. CrossRef Medline

Kenet T, Bibitchkov D, Tsodyks M, Grinvald A, Arieli A (2003) Spontaneously emerging cortical representations of visual attributes. Nature 425: 954-956. CrossRef Medline

Kleiner M, Brainard D, Pelli D (2007) What's new in Psychtoolbox-3? Perception 36 .

Kohn A, Smith MA (2005) Stimulus dependence of neuronal correlation in primary visual cortex of the macaque. J Neurosci 25:3661-3673. CrossRef Medline

de la Rocha J, Doiron B, Shea-Brown E, Josíc K, Reyes A (2007) Correlation between neural spike trains increases with firing rate. Nature 448:802806. CrossRef Medline

Lee D, Port NL, Kruse W, Georgopoulos AP (1998) Variability and corre- 
lated noise in the discharge of neurons in motor and parietal areas of the primate cortex. J Neurosci 18:1161-1170. Medline

Mitchell JF, Sundberg KA, Reynolds JH (2009) Spatial attention decorrelates intrinsic activity fluctuations in macaque area V4. Neuron 63:879888. CrossRef Medline

Morita K, Kalra R, Aihara K, Robinson HP (2008) Recurrent synaptic input and the timing of gamma-frequency-modulated firing of pyramidal cells during neocortical "UP” states. J Neurosci 28:1871-1881. CrossRef Medline

Nir Y, Mukamel R, Dinstein I, Privman E, Harel M, Fisch L, Gelbard-Sagiv H, Kipervasser S, Andelman F, Neufeld MY, Kramer U, Arieli A, Fried I, Malach R (2008) Interhemispheric correlations of slow spontaneous neuronal fluctuations revealed in human sensory cortex. Nat Neurosci 11:1100-1108. CrossRef Medline

Nordhausen CT, Maynard EM, Normann RA (1996) Single unit recording capabilities of a 100 microelectrode array. Brain Res 726:129-140. CrossRef Medline

Okun M, Lampl I (2008) Instantaneous correlation of excitation and inhibition during ongoing and sensory-evoked activities. Nat Neurosci 11:535-537. CrossRef Medline

Perkel DH, Gerstein GL, Moore GP (1967) Neuronal spike trains and stochastic point processes. II. Simultaneous spike trains. Biophys J 7:419-440. CrossRef Medline

Poort J, Roelfsema PR (2009) Noise correlations have little influence on the coding of selective attention in area V1. Cereb Cortex 19:543-553. CrossRef Medline

Poulet JF, Petersen CC (2008) Internal brain state regulates membrane potential synchrony in barrel cortex of behaving mice. Nature 454:881-885. CrossRef Medline

Rasch MJ, Schuch K, Logothetis NK, Maass W (2011) Statistical comparison of spike responses to natural stimuli in monkey area V1 with simulated responses of a detailed laminar network model for a patch of V1. J Neurophysiol 105:757-778. CrossRef Medline

Roe AW, Chelazzi L, Connor CE, Conway BR, Fujita I, Gallant JL, Lu H, Vanduffel W (2012) Toward a unified theory of visual area v4. Neuron 74:12-29. CrossRef Medline

Rotermund D, Taylor K, Ernst UA, Kreiter AK, Pawelzik KR (2009) Attention improves object representation in visual cortical field potentials. J Neurosci 29:10120-10130. CrossRef Medline

Rousche PJ, Normann RA (1992) A method for pneumatically inserting an array of penetrating electrodes into cortical tissue. Ann Biomed Eng 20: 413-422. CrossRef Medline

Samonds JM, Potetz BR, Lee TS (2009) Cooperative and competitive interactions facilitate stereo computations in macaque primary visual cortex. J Neurosci 29:15780-15795. CrossRef Medline

Shadlen MN, Movshon JA (1999) Synchrony unbound: a critical evaluation of the temporal binding hypothesis. Neuron 24:67-77, 111-125. CrossRef Medline
Shadlen MN, Newsome WT (1998) The variable discharge of cortical neurons: implications for connectivity, computation, and information coding. J Neurosci 18:3870-3896. Medline

Shoham S, Fellows MR, Normann RA (2003) Robust, automatic spike sorting using mixtures of multivariate $t$-distributions. J Neurosci Methods 127:111-122. CrossRef Medline

Smith MA, Kohn A (2008) Spatial and temporal scales of neuronal correlation in primary visual cortex. J Neurosci 28:12591-12603. CrossRef Medline

Smith MA, Jia X, Zandvakili A, Kohn A (2013) Laminar dependence of neuronal correlations in visual cortex. J Neurophysiol 109:940-947. CrossRef Medline

Sommer MA, Wurtz RH (2000) Composition and topographic organization of signals sent from the frontal eye field to the superior colliculus. J Neurophysiol 83:1979-2001. Medline

Suner S, Fellows MR, Vargas-Irwin C, Nakata GK, Donoghue JP (2005) Reliability of signals from a chronically implanted, silicon-based electrode array in non-human primate primary motor cortex. IEEE Trans Neural Syst Rehabil Eng 13:524-541. CrossRef Medline

Tanigawa H, Lu HD, Roe AW (2010) Functional organization for color and orientation in macaque V4. Nat Neurosci 13:1542-1548. CrossRef Medline

Taylor K, Mandon S, Freiwald WA, Kreiter AK (2005) Coherent oscillatory activity in monkey area $\mathrm{v} 4$ predicts successful allocation of attention. Cereb Cortex 15:1424-1437. CrossRef Medline

Thiele A, Stoner G (2003) Neuronal synchrony does not correlate with motion coherence in cortical area MT. Nature 421:366-370. CrossRef Medline

Traub RD, Kopell N, Bibbig A, Buhl EH, LeBeau FE, Whittington MA (2001) Gap junctions between interneuron dendrites can enhance synchrony of gamma oscillations in distributed networks. J Neurosci 21:9478-9486. Medline

Tsodyks M, Kenet T, Grinvald A, Arieli A (1999) Linking spontaneous activity of single cortical neurons and the underlying functional architecture. Science 286:1943-1946. CrossRef Medline

Ungerleider LG, Galkin TW, Desimone R, Gattass R (2008) Cortical connections of area V4 in the macaque. Cereb Cortex 18:477-499. CrossRef Medline

Yoshioka T, Levitt JB, Lund JS (1992) Intrinsic lattice connections of macaque monkey visual cortical area V4. J Neurosci 12:2785-2802. Medline

Zhou Z, Bernard MR, Bonds AB (2008) Deconstruction of spatial integrity in visual stimulus detected by modulation of synchronized activity in cat visual cortex. J Neurosci 28:3759-3768. CrossRef Medline

Zohary E, Shadlen MN, Newsome WT (1994) Correlated neuronal discharge rate and its implications for psychophysical performance. Nature 370:140-143. CrossRef Medline 